\title{
No measurable adverse effects of Lassa, Morogoro and Gairo arenaviruses on their rodent reservoir host in natural conditions
}

\author{
Joachim Mariën ${ }^{1 *}$, Benny Borremans ${ }^{1,5}$, Sophie Gryseels ${ }^{1,6}$, Barré Soropogui' ${ }^{2}$, Luc De Bruyn ${ }^{1}$, \\ Gédéon Ngiala Bongo ${ }^{3,7}$, Beate Becker-Ziaja ${ }^{3}$, Joëlle Goüy de Bellocq ${ }^{4}$, Stephan Günther ${ }^{3}$, N'Faly Magassouba ${ }^{2}$, \\ Herwig Leirs ${ }^{1}$ and Elisabeth Fichet-Calvet ${ }^{3}$
}

\begin{abstract}
Background: In order to optimize net transmission success, parasites are hypothesized to evolve towards causing minimal damage to their reservoir host while obtaining high shedding rates. For many parasite species however this paradigm has not been tested, and conflicting results have been found regarding the effect of arenaviruses on their rodent host species. The rodent Mastomys natalensis is the natural reservoir host of several arenaviruses, including Lassa virus that is known to cause Lassa haemorrhagic fever in humans. Here, we examined the effect of three arenaviruses (Gairo, Morogoro and Lassa virus) on four parameters of wild-caught Mastomys natalensis: body mass, head-body length, sexual maturity and fertility. After correcting for the effect of age, we compared these parameters between arenaviruspositive (arenavirus RNA or antibody) and negative animals using data from different field studies in Guinea (Lassa virus) and Tanzania (Morogoro and Gairo viruses).
\end{abstract}

Results: Although the sample sizes of our studies (1297, 749 and 259 animals respectively) were large enough to statistically detect small differences in body conditions, we did not observe any adverse effects of these viruses on Mastomys natalensis. We did find that sexual maturity was significantly positively related with Lassa virus antibody presence until a certain age, and with Gairo virus antibody presence in general. Gairo virus antibody-positive animals were also significantly heavier and larger than antibody-free animals.

Conclusion: Together, these results suggest that the pathogenicity of arenaviruses is not severe in M. natalensis, which is likely to be an adaptation of these viruses to optimize transmission success. They also suggest that sexual behaviour might increase the probability of $M$. natalensis to become infected with arenaviruses.

Keywords: Arenavirus, Lassa virus, Morogoro virus, Gairo virus, Mastomys natalensis, Rodent-borne disease, Hostpathogen interaction, Reservoir host

\section{Background}

The Natal multimammate mouse, Mastomys natalensis (Smith, 1834), is the natural host of six known Old World arenaviruses (Arenaviridae, Mammarenavirus): Lassa (LASV) and a Mobala-like virus in West Africa, and Morogoro (MORV), Mopeia, Luna and Gairo viruses (GAIV) in Southern East Africa [1-7]. Of these six viruses, only LASV is known to cause Lassa haemorrhagic fever in humans, who can become infected by close contact with infectious

\footnotetext{
* Correspondence: Joachim.marien@uantwerpen.be

${ }^{1}$ Evolutionary Ecology Group, University of Antwerp, Antwerp, Belgium Full list of author information is available at the end of the article
}

rodents or their excretions [1]. Annually around 200,000 people are affected, with a fatality rate of $1-2 \%$ [8-10].

While the pathogenic effects of LASV on humans are well-documented [11, 12], little attention has been paid to the effects on its reservoir host. Nevertheless, knowledge of how parasites affect their reservoir hosts is relevant in the study of wildlife diseases, as this helps to understand how host population dynamics or behaviour affect the spread of the parasite through the population [13]. In general, in order to optimize net transmission success, parasites face a trade-off between within-host reproduction, which requires the utilisation of host 
resources, and between-host transmission probability, which requires the hosts to be sufficiently healthy and long-lived to encounter new susceptible individuals [14-16]. Usually, a long evolutionary history in a particular host results in low virulence towards the natural reservoir $[17,18]$.

Most information about arenavirus pathogenicity is derived from inoculation studies on laboratory house mice (Mus musculus) with lymphocytic choriomeningitis virus (LCMV), an Old World arenavirus that shares 60\% amino acid similarity with LASV [19]. House mice are the reservoir hosts of LCMV and most LCMV strains result in symptom-free infection, despite high levels of viral growth $[20,21]$. Nevertheless, severe disease (including meningitis, reduced growth and death) did develop for some mouse breed - LCMV strain combinations [22-25]. Variation in pathogenicity was also noted in vesper mice (Calomys callosus and Calomys musculinus) which are the natural hosts of respectively New World Machupo and Junin arenaviruses. Inoculation of C. callosus with Machupo virus resulted in a dual response in which about half of the infected animals became immunocompetent with transient viremia and no disease symptoms, whereas the other half became immunotolerant and exhibited persistent viremia and reduced body size, fertility and lifespan [26]. Inoculation with Junin virus caused a similar response in C. musculinus, where adults remained asymptomatic while juveniles experienced reduced body size, fertility and lifespan $[27,28]$.

Few studies have examined the effects of arenaviruses in M. natalensis. Two inoculation studies, on LASV and MORV, showed no or only mild overt disease symptoms in their rodent host $[29,30]$. MORV inoculations in adult wild type $M$. natalensis caused significant temporary $7 \%$ weight loss 10 days post-infection in about half of the animals. Although these animals recovered completely in the laboratory, such weight loss in natural circumstances might have significant consequences for survival probability. In contrast to MORV inoculation studies, two field studies found that LASV reduces growth and fecundity of $M$. natalensis, and suggested that LASV severely affects its rodent host [31, 32]. These studies should however be interpreted with caution, as the first provided limited statistical proof, while the latter used body mass category as an estimate to correct for an age effect on prevalence, which is a potentially unreliable proxy [33].

Knowledge of the exact age is essential to estimate pathogenicity effects, since age correlates with many morphometric and sexual parameters. More importantly, in case of acute infections with long-term presence of antibodies (observed for LASV-related arenaviruses in $M$. natalensis [2, 34, 35]), older animals are more likely to have already encountered the infection, and developed antibodies, during their lifetime simply due to the longer time window they have been alive. In this situation, the probability of detecting active, recent infection decreases with age, as more susceptible (antibody-negative) animals are available in the younger age categories compared to the older categories [35]. Therefore, if one wants to investigate the effect of infection on an age-dependent host variable (e.g. body mass, head-body length or sexual maturity), the effect of age should be disentangled from that of the variable [36].

In this paper, we want to systematically analyse data from three different $M$. natalensis-borne arenaviruses in the same way, in order to confidently test whether infection affects the host in natural conditions. We will test the relationships between arenavirus (LASV, MORV and GAIV) infection and body mass, body length, sexual maturity and fertility. We correct for the effects of age by using eye lens weight (ELW), which is known to be a good proxy for age because the eye lens is continuously growing, independent of environmental factors [37-41].

We previously investigated the relationships between LASV infection and several parameters such as village, habitat, season, age and abundance in Guinea [42-44]. Using more recent data, we here expand this analysis to body condition and increase the dataset from 553 to 1,297 individuals. For MORV, a previous investigation on its effect on $M$. natalensis body condition was performed on a small dataset $(n=171)$ during one season and locality only, where no significant relationship between MORV infection and body mass index was found, although a model including body mass index did result in the best prediction of active MORV infection [35]. Here we supplement this dataset with new data in order to arrive at a sample size of 749 individuals. Our recent work on GAIV tested the relationship between infection and host condition (using infection status as dependent variable) [2], but here we re-analyse these data in exactly the same way as the other two viruses (using body condition as dependent variable), as this allows a direct comparison between the three viruses.

\section{Methods \\ Guinean study sites, rodent sampling, and LASV screening of rodents}

For the examination of the relationships between LASV and $M$. natalensis body condition, we re-analysed data from previous publications [42, 44, 45] and also present new data. Here, we provide a summary of the data collection procedures, but refer to the mentioned publications for more details. Rodents were captured in thirteen villages with reported human LASV cases in Upper Guinea during an intermittent time span between 2003 and 2015. Traps were placed both indoors and outdoors during the rainy and dry season. Captured rodents were 
humanely killed and measured morphometrically. Sexual maturity of females was determined by pregnancy, lactation status and by signs of scars in a large uterus (width $>4 \mathrm{~mm}$ ). Body mass of pregnant females was adjusted for foetus + uterus mass. Fertility of pregnant females was determined by counting the number of foetuses inside the uterus. Males were considered sexually mature if the vesiculae seminales were swollen and their surface exceeded $100 \mathrm{~mm}^{2}$. Eyes were preserved in $10 \%$ formalin, the lenses were extracted, cleaned and dried for $2 \mathrm{~h}$ at $100{ }^{\circ} \mathrm{C}$, and then weighed to the nearest $0.1 \mathrm{mg}$. Whole blood, spleen, kidney, and liver were collected during the autopsies and stored in liquid nitrogen or in $-20{ }^{\circ} \mathrm{C}$. Detection of LASV viral RNA (vRNA) was performed and examined by RT-PCR on blood and spleen of $1,298 \mathrm{M}$. natalensis $[46,47]$. Detection of anti-LASV antibodies was performed on 1,139 M. natalensis and examined by indirect immunofluorescence assay [48, 49]. For this assay, Vero cells infected with LASV strain Bantou were spread on immunofluorescence slides, air dried, and acetone-fixed. Antibodies in positive samples would then bind to antigens presented by the Vero cells and be visualized with anti-mouse IgG-FITC secondary antibodies. In total, 168 (13\%) LASV vRNA and 318 (28\%) antibody positive individuals were detected.

\section{Tanzanian study sites, rodent sampling, and MORV or GAIV screening of rodents}

For the examination of the relationships between MORV or GAIV infection and $M$. natalensis body condition, we (re-)analysed data from previous publications [2, 3, 35] and here present new antibody data from the nine localities where MORV circulates as described in [3]. Rodents were trapped outdoors in the Morogoro region in Tanzania intermittently between 2008 and 2012. Captured rodents were humanely killed and identified morphometrically. Reproductive status was measured by investigating external characteristics of the reproductive organs, as described in [39]. Eye lens weight (ELW) was measured using the method described for the LASV studies, except that they were dried for $>2 \mathrm{~h}$ at $80{ }^{\circ} \mathrm{C}$. Organs were preserved in RNAlater for viral detection, and blood on serobuvard filter paper $( \pm 15 \mu \mathrm{l} /$ punch; Serobuvard, LDA 22, Zoopole, France) for antibody detection. In total, 743 and $259 \mathrm{M}$. natalensis were tested for respectively MORV and GAIV vRNA using RT-PCR reaction [5, 46], and 683 and $259 M$. natalensis were tested for the presence of anti-MORV and antiGAIV antibodies using indirect immunofluorescence assay. Vero cells infected with MORV strain 3017/2004 were spread on immunofluorescence slides, air dried, acetone-fixed, and visualised as described for LASV. For MORV and GAIV respectively, 48 (6\%) and 29 (11\%) animals tested vRNA positive, and $73(11 \%)$ and $25(9 \%)$ animals tested antibody positive [2, 3, 35].

\section{Statistical analysis}

We tested the relationships between arenavirus infection and four different measures of $M$. natalensis' body condition: body mass, head-body (HB) length and sexual maturity for all viruses, and fertility for LASV only with linear mixed models and generalised linear mixed models. For all the investigated parameters it was important to disentangle the effect of age from that of the measured variable. For this reason, we developed models with one of the parameters of interest as dependent variable and ELW (proxy for age), infection status (RNA/antibody positive or negative) and their interaction as independent fixed variables. We also included sex, season (rainy/dry) and their interaction with ELW and infection status as independent fixed variables, and year and village as independent random variables to correct for effects of year and location. We log-transformed body mass, HB length and ELW since we observed a linear relation between log(body mass) and $\log (\mathrm{HB}$ length) on the one hand and $\log (\mathrm{ELW})$ on the other hand. The relationships to sexual maturity (i.e. the probability of being sexually active, $1=$ active, $0=$ inactive) were assessed assuming a binomial distribution (logit-link function). The relationships to fertility (i.e. number of fetuses inside the uterus, only for sexually mature females) were assessed assuming a Poisson distribution (log-link function). We performed separate analyses for three different infection statuses: (i) only arenavirus RNA, (ii) only antibody, and (iii) either arenavirus RNA or antibody presence, or both.

In one of the 10 villages investigated in 2013 in Upper Guinea, no LASV was detected. We therefore compared the effect of infection in $M$. natalensis living in this LASV negative village (Tambaya, $n=50$ ) to those living in the nearest LASV positive village (Brissa, $n=62$ ). Season and year were removed from this model as these animals were collected in the same period, and LASV village (positive/negative) was implemented as an independent fixed variable.

We used the lmer and glmer functions of the lme4 package (version 1.1-7) of the $\mathrm{R}$ statistical software version 3.3.0 [50] to run the mixed models. When fitting the models, we started with the fully parameterised models (all interactions between the independent fixed variables) and sequentially dropped variables that had the highest insignificant p-values. Significance was tested with the likelihood ratio test (assuming a $\chi^{2}$ residual distribution).

\section{Results}

Because the effects of the parameters ELW, infection status and their interactions on the body condition 
variables (body mass, head-body length, sexual maturity and fertility) were of main interest in this study, we summarized these in Table 1. The effects of other parameters (sex, season and their interaction) on the body condition variables, were presented in Additional file 1: Tables S1 and S2).

The three body condition predictor variables (body mass, head-body length and sexual maturity) were all highly correlated with age in the three investigated populations of M. natalensis $\left(\chi_{1}^{2}>25.90, P<0.01\right)$ (Figs. 1,2 and 3). As reported previously, presence of either vRNA or antibody is also correlated significantly with age for all three viruses $\left(\chi_{1}^{2}>4.75, P<0.03\right)$ (Additional file 1: Table S3).

Correcting for this general age effect, we observed almost no effect of either active or past arenavirus infection on body condition (Table 1). For none of the investigated viruses, presence of vRNA was significantly related to any of the predictor variables, nor were any of the interactions between vRNA presence and ELW significantly related to any of the predictor variables (Table 1).

Antibody presence was not significantly related to any of the predictor variables for MORV. For GAIV, we found significant relationships between antibody presence and all three body condition predictor variables (Table 1, Fig. 3): antibody-positive $M$. natalensis were significantly heavier $\left(X_{1}^{2}=13.28, P<0.01\right)$ and larger $\left(X_{1}^{2}\right.$ $=8.45, P<0.01)$ than antibody negative ones, and had a significantly higher probability of being sexually mature $\left(X_{1}^{2}=8.57, P<0.01\right)$. For LASV, antibody presence alone was not significantly related to body mass and $\mathrm{HB}$ length. The relationship between LASV antibodies and sexual maturity could not be assessed due to a significant interaction between antibody and ELW $\left(\chi_{1}^{2}=5.42\right.$, $P=0.02$ ), in which young antibody-positive $M$. natalensis had a higher probability of being sexually mature than antibody-negative animals (Fig. 1). This difference disappeared for older animals where more animals are sexually mature $\left(X_{1}^{2}=1.86, P=0.06\right)$. Interactions between antibody presence and ELW had no significant effect on any of the body condition variables in the case of MORV and GAIV.

The presence of either vRNA, antibody or both (indicative of successful infection at any point in the past) was not significantly related to any of the predictor variables for MORV and LASV, nor was the interaction between this definition of infection status and ELW significantly related to any of the predictor variables. For GAIV, there were significant relationships between the infection status and all three body condition predictor variables: past or recent infected animals where significantly heavier $\left(X_{1}^{2}=8.60\right.$, $P<0.01)$ and larger $\left(\chi_{1}^{2}=6.23, P=0.01\right)$, and had a higher probability of being sexually mature $\left(\chi_{1}^{2}=6.14, P=0.01\right)$ than animals that were never infected (Table 1, Fig. 3).
We could analyse the relationship between infection and fertility for the LASV dataset only. Fertility in females as measured by the number of foetuses was independent of age $\left(X_{1}^{2}=1.84, P=0.17\right)$, and no significant difference in number of foetuses was observed between LASV RNA/antibody-positive and negative pregnant females $\left(X_{1}^{2}<2.14, P>0.14\right)$ (Table 1$)$. For LASV, we could also compare a LASV-negative village (Tambaya) with its nearest LASV-positive village (Brissa). We did not find any significant differences in body mass, head-body length or sexual maturity between $M$. natalensis in the two villages $\left(X_{1}^{2}<2.62, P>0.09\right)$ (Additional file 1 : Table S4; Additional file 2: Figure S1).

\section{Discussion}

We did not observe any significant negative associations between arenavirus infection and M. natalensis morphometric or sexual parameters, suggesting that these viruses are harmless for the reservoir hosts in natural conditions. Our results are in contrast with the studies of Demartini et al. [31] and Lalis et al. [32]. Demartini et al. [31] found that LASV infections resulted in less heavy animals and inflammatory lesions. As these authors noted, important limitations of their study were the small sample size $(n=28)$ and lack of information about the animals' age. While Lalis et al. [32] also found no association between LASV presence and host body mass, they did find that LASV positive animals had a significantly smaller body size, skull and size of the reproductive organs in the adult category. Their adult category based on the body mass (>40 g) was highly variable and can cover a range of 40-97 $\mathrm{g}$ (ELW $=15-38 \mathrm{mg}$ in figure 5 in EFC et al. [43]). Among this body weight class, 73\% (47/64) of the LASV positive animals were belonging to the youngest ELW class $(15-25 \mathrm{mg})$. We can suggest that their 30 LASV positive adult samples were possibly younger, smaller and more inactive sexually than their 38 LASV negative ones.

Our results are consistent with the laboratory inoculation studies of Walker et al. [30] and Borremans et al. [29], who observed no or only mild disease symptoms of arenaviruses in M. natalensis. Walker et al. [30] showed that neonatal animals inoculated with LASV exhibited a persistent tolerant infection with no histopathological signs of disease. Nevertheless, some animals infected as adults developed moderate (not deadly) meningoencephalitis. Borremans et al. [29] found that inoculation of $M$. natalensis with MORV could decrease the weight of animals between days 7 and 15 post-infection $( \pm 7 \%$ of normal weight in $40 \%$ of animals), but this decrease was only temporary and did not further affect the animals' growth rates. 


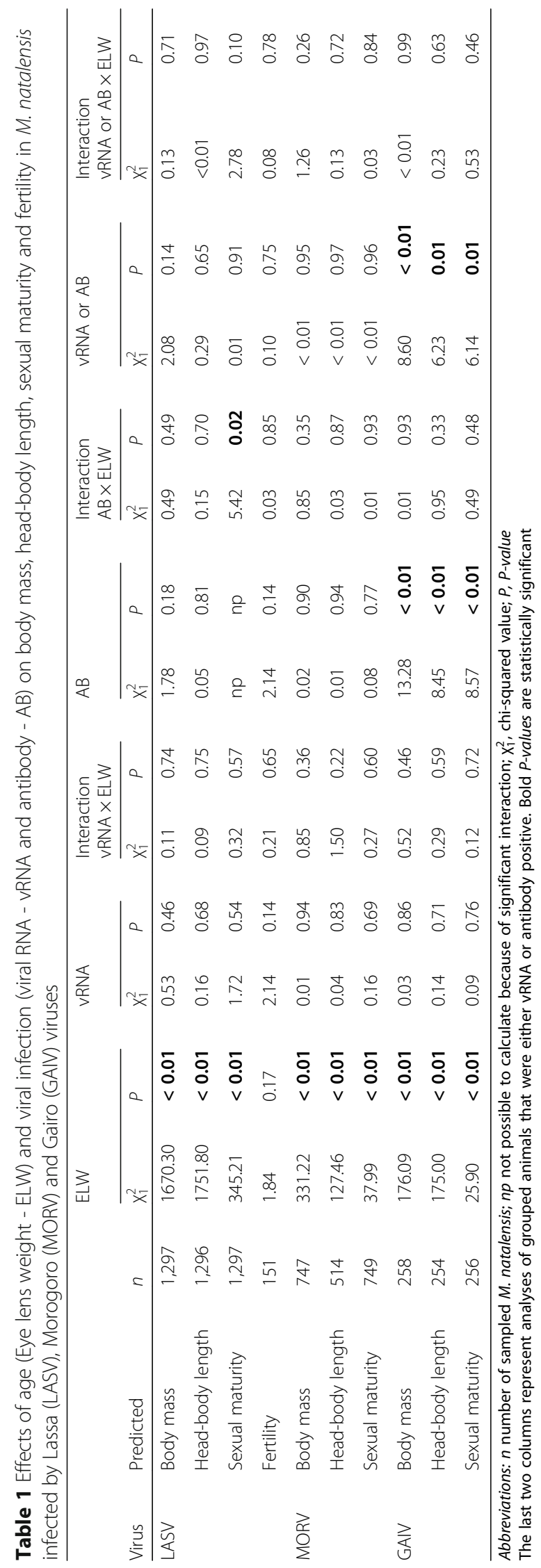



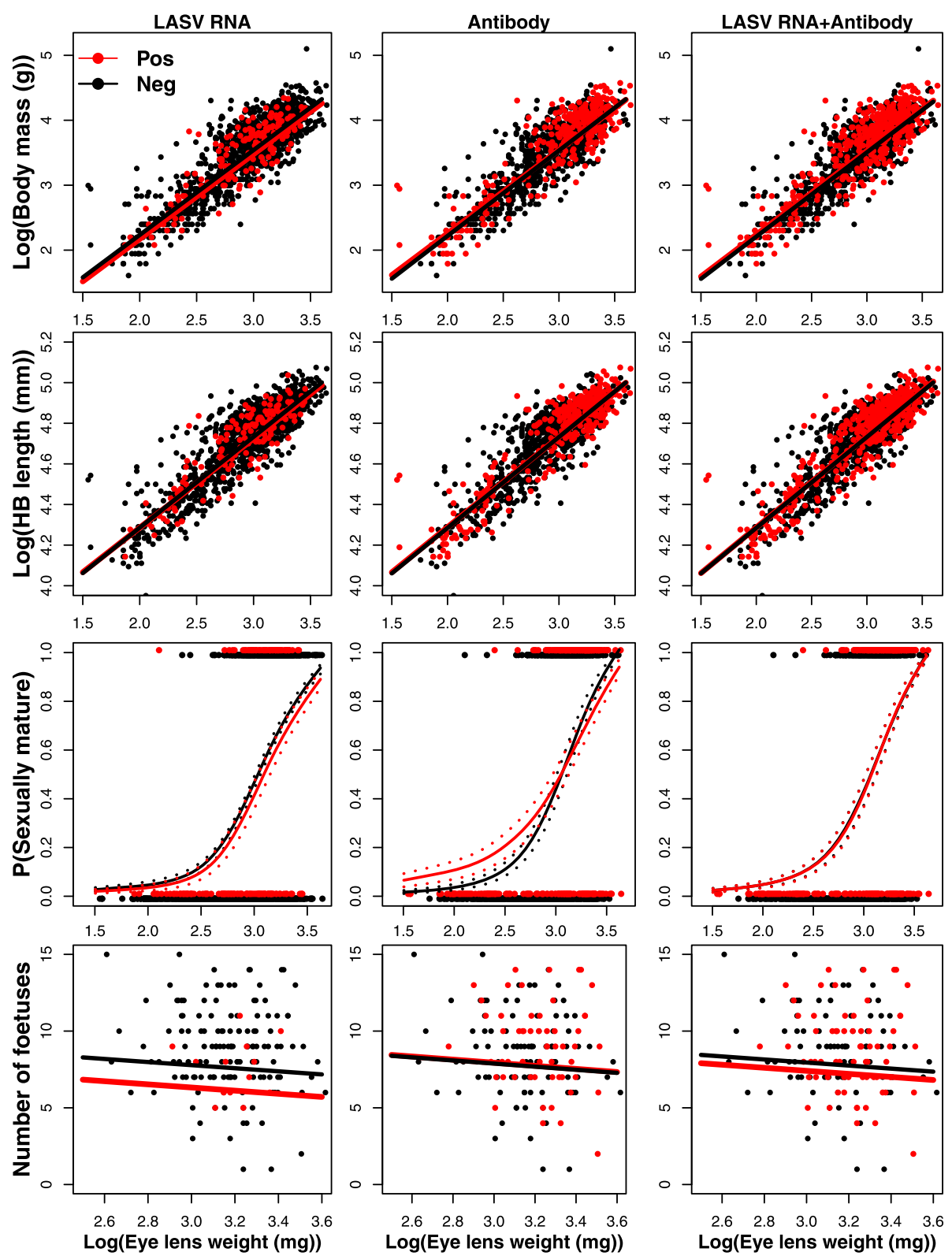

Fig. 1 Relationships between body mass, head-body length, sexual maturity ( $1=$ active and 0 =inactive), fertility (\# foetuses) and eye lens weight (as a proxy for age) in M. natalensis infected by Lassa virus. Red=positive, black= negative. Dashed lines represent standard errors on the predicted probabilities

It should be noted that we did not provide proof that these viruses never affect their reservoir host. For example, our data do not exclude that some animals become sick, die quickly from disease and thus are not trapped during field studies. Also, we only checked four host parameters, while viruses might affect their host in many different ways (e.g. differences in hormone levels, brain size or temperature may be found [21]). Such complex relationships between parasite infection and host fitness were indeed observed in other rodent-borne diseases. For example, infection of Puumala hantavirus in bank voles (Myodes glareolus) was initially assumed to be asymptomatic [51-53] but recent long-term field studies observed negative effects on the survival probability and an equivocal impact on the fecundity of females $[36,54,55]$. An important difference between Old World arenaviruses and hantaviruses, however, is that the latter causes persistent infections in their reservoir hosts [56], while the former infects their hosts mainly acutely $[29,43]$. The lower survival probability caused by hantaviruses could therefore perhaps be explained by an accumulation of small deleterious effects over time, which 


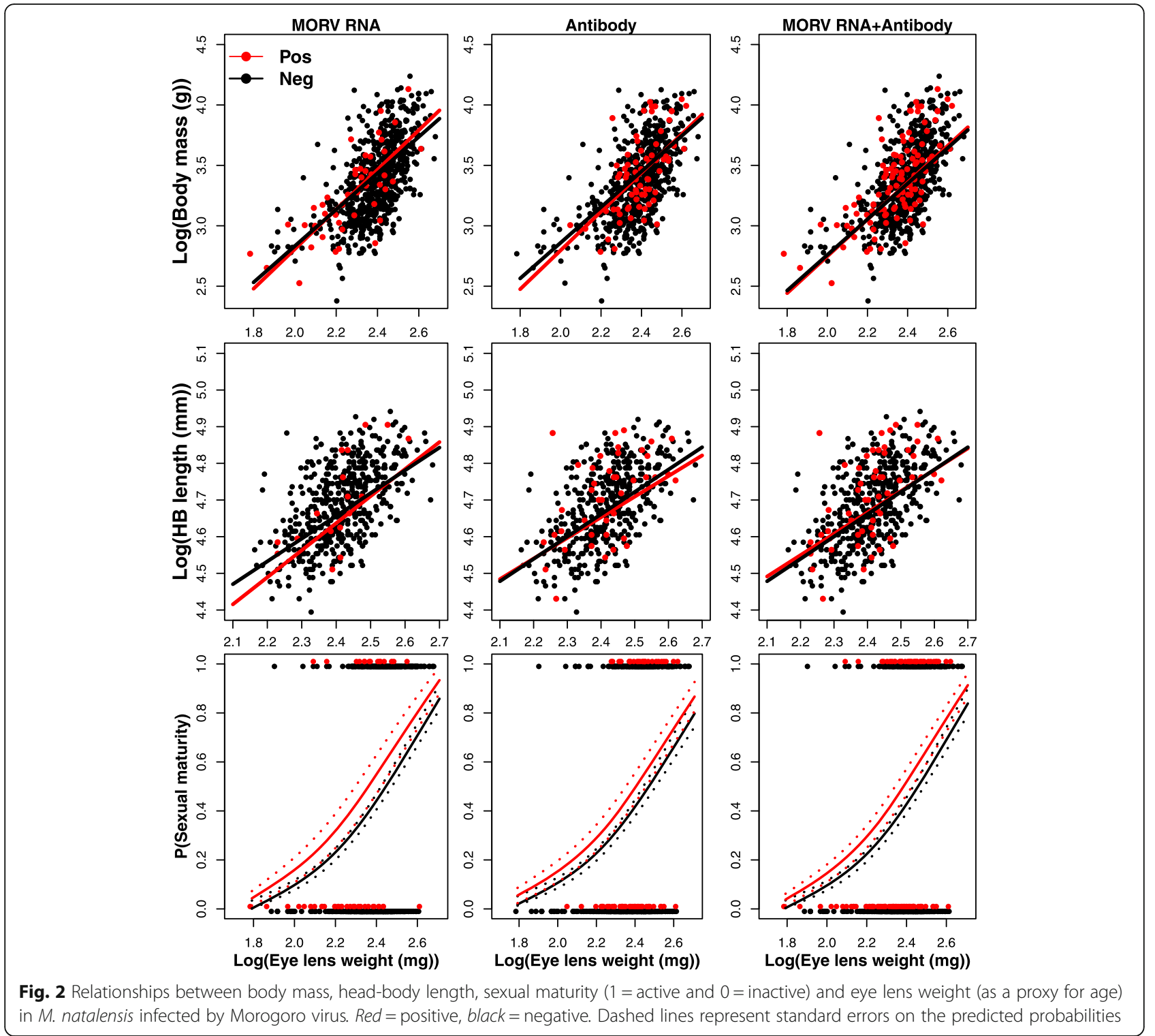

might not be the case during the relatively short arenavirus infections. Nevertheless, performing survival analyses would be a logical next step to further explore possible effects of arenaviruses in M. natalensis, and could be done by investigating long-term capturemark-recapture data.

In general, our study supports the hypothesis that the pathogenicity of arenaviruses is low in their natural hosts. This is likely an adaptation of these viruses to increase the probability of transmitting the infection to other individuals. In contrast, highly pathogenic viruses might fail in transmission because they reduce the host's infectious period, or reduce its contact rate as a tradeoff for energy to eradicate the virus [13, 57]. A non-pathogenic lifestyle would be especially beneficial for MORV, since MORV is endemic in populations of
M. natalensis that fluctuate heavily between seasons, generally ranging from 20 to 300 individuals per hectare $[5,58]$. Mathematical models suggest that these fluctuations impose a severe impediment for MORV persistence, because during the low density periods, the transmission chain is heavily dependent on a few susceptible and infectious individuals only [59]. Nevertheless, MORV can persist in these highly fluctuating populations, and can be detected even at very low densities [35]. In order to survive these bottlenecks, we assume that adaptation has driven MORV to a low-pathogenic lifestyle. A similar situation is suggested for the persistence of Junin arenavirus in populations of Calomys musculinus. Vitullo \& Merani [27, 60] found that Junin virus did not affect the fitness of adult $C$. musculinus and, based on a mathematical model, they assumed that 


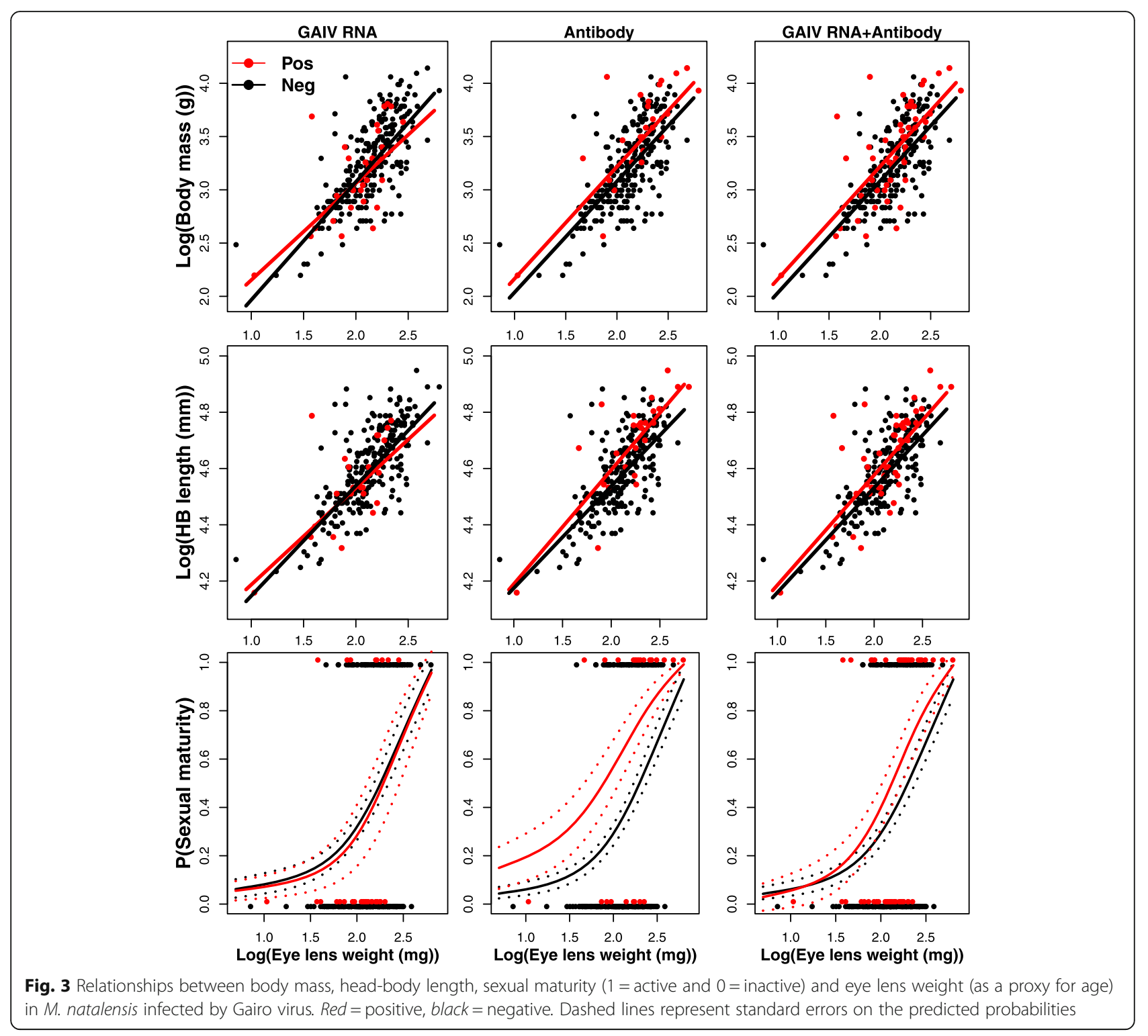

these unaffected animals are a prerequisite for viral persistence.

After correcting for the effect of age, we found that GAIV antibody-positive $M$. natalensis were significantly heavier, larger and reached sexual maturity earlier than antibody-free rodents. We also found that LASV antibody-positive rodents reached sexual maturity earlier until a certain age. These results are compatible with the hypothesis that sexual behaviour increases the probability of becoming infected with arenaviruses, and that sexual transmission could therefore be an important mechanism for the spread of arenaviruses in populations of $M$. natalensis. Although this relationship was not found for LASV in older animals or for MORV at all, sexual transmission would indeed be a highly beneficial transmission route for arenaviruses. Arenaviruses are assumed to be transmitted horizontally (at least partly) by as yet unconfirmed pathways [2, 35, 42, 44], and $M$. natalensis has a promiscuous mating system in which both males and females endeavour to copulate with numerous mates $[39,61,62]$. Sexual transmission is also assumed to be important in other virus-rodent host systems. Cage experiments showed that the transmission rate of Machupo arenavirus in C. callosus was considerably higher in opposite sex pairs than in same sex pairs, and high concentrations of virus and antibody were found in the reproductive organs [26]. In natural populations of bank voles infected with Puumala hantavirus, seroconversion was observed to occur more frequently during the reproductive season and in sexually active individuals [51]. However, a recent long-term field study showed that seroconversion rates of bank voles are 
significantly higher outside the reproductive season, which occurs in winter [63]. This last result supports the hypothesis of an active indirect transmission through contaminated soil during wet and cold conditions [64]. The two conflicting results highlight that patterns within the same parasite-host system can differ considerably due to different environmental conditions.

Otherwise, the observed relation between antibodies and sexual maturity in our study might also be explained by the hypothesis that arenavirus replication enhances sexual maturity or behaviour of its host. This might be likely, as it has been shown that LCMV can severely disrupt hormonal regulation in laboratory mice $[25,65,66]$. Similarly, Kallio et al. [36] suggest that infection with Puumala hantavirus enhances breeding in young female bank voles, but not in old ones.

\section{Conclusion}

We do not find any evidence that $M$. natalensis-borne arenaviruses negatively affect their natural host in natural circumstances. While adverse effects that lead to low capture probability in a subset of the infected population cannot be ruled out, our results suggest that the pathogenicity of arenaviruses is low in M. natalensis in general. This implies that these viruses do not significantly affect the population dynamics of $M$. natalensis. Furthermore, as our study indicates that sexual behaviour can be important for arenavirus transmission in natural populations of $M$. natalensis, it would be interesting to determine the extent to which sexual transmission occurs. If an important mode of transmission, and considering mating frequency could be expected to be density-dependent in $M$. natalensis, control measures aimed at reducing the abundance of rodents during the breeding season may be highly effective for virus eradication.

\section{Additional files}

Additional file 1: Table S1. Effects of sex and viral infection (viral RNA VRNA and antibody - AB) on body mass, head-body length, sexual maturity and fertility in M. natalensis infected by Lassa (LASV), Morogoro (MORV) and Gairo (GAIV) viruses. Estimate effects were shown on a log-scale to allow linear comparison. The last two columns represent animals that were vRNA or antibody positive. ( $n=$ number of sampled M. natalensis; *statistically significant, NP, not possible to calculate because of significant interaction with ELW). Table S2. Effects of season and viral infection (viral RNA - vRNA and antibody - AB) on body mass, head-body length, sexual maturity and fertility in M. natalensis infected by Lassa (LASV), Morogoro (MORV) and Gairo (GAIV) viruses. Estimate effects were shown on a log-scale to allow linear comparison. The last two columns represent animals that were vRNA or antibody positive. ( $n$ = number of sampled M. natalensis; ${ }^{*}$ statistically significant, NPa, not possible to calculate because of significant interaction with ELW, NP' , not possible to calculate because there were not enough data sampled in one of the two seasons). Table S3. Relation between viral infection (viral RNA - vRNA and antibody - AB) and age (eye lens weight) in M. natalensis infected by Lassa (LASV), Morogoro (MORV) and Gairo (GAIV) viruses. Table S4. Effects on body mass, head-body length and sexual maturity of $M$. natalensis between a Lassa virus (LASV) positive and negative village (Brissa versus Tambaya) $(n=$ number of sampled M. natalensis). (DOCX $27 \mathrm{~kb}$ )

Additional file 2: Figure S1. Relationships between body mass, headbody length, sexual maturity ( $1=$ active and $0=$ inactive) and Eye lens weight (as a proxy for age) in M. natalensis infected by Lassa virus (LASV). Red $=$ LASV positive village (Brissa), black = LASV negative village (Tambaya). Dashed lines represent standard errors on the predicted probabilities. (PDF $100 \mathrm{~kb}$ )

\section{Abbreviations}

ELW: Eye lens weight; GAIV: Gairo virus; HB: Head body; LASV: Lassa virus; LCMV: Lymphocytic choriomeningitis virus; MORV: Morogoro virus; vRNA: viral RNA

\section{Acknowledgements}

We thank Fodé Kourouma, Amara Bongo, Amadou Doré and Fanta Bérété from the Projet des Fièvres Hémorragiques de Guinée and the staff (in particular Khalid Kibwana and Shabani Lutea) at the Pest Management Centre in Morogoro (Tanzania) for their excellent support in the field. We are grateful to Nathalie Van Houtte of the University of Antwerp for laboratory technical support.

\section{Funding}

This work was supported by the University of Antwerp and the Antwerp study centre for disease (ASCID) grant number GOA BOF FFB3567, the INCO-DEV grant ICA4-CT2002-10050, the Marie Curie fellowship to EFC (PIEF-GA-2009235164), grants FI 1781/1-1, GU 883/3-1 and LE SPP from the German Research Foundation (Focus Programs 1596 from Deutsche Forschungsgemeinschaft), BEBUC (Bourse d'Excellence Bringmann aux Universités Congolaises) and Holger-Pöhlmann Foundation (Germany). Joachim Mariën is a research fellow of the Flemish Interuniversity Council (VLIR-UOS). Benny Borremans and Sophie Gryseels were research fellows of Research Foundation Flanders (FWO) during part of the study.

\section{Availability of data and materials}

The datasets used and/or analysed during the current study available from the corresponding author on reasonable request. Voucher specimens of all individuals analysed for Morogoro and Gairo virus are maintained at the Evolutionary Ecology group at the University of Antwerp.

\section{Authors' contributions}

Conceived the study: JM and EF-C. Wrote the paper: JM, BB and SGr. Performed the experiments: BB., SGr, BS, GNB, BB-Z, JG-B and EF-C. Performed the analyses: JM and LDB. Supervised field and laboratory work: JG-B, SGu, $\mathrm{HL}$, NM and EF-C. All authors read and approved the final manuscript.

\section{Competing interests}

The authors declare that they have no competing interests.

\section{Consent for publication}

Not applicable.

\section{Ethics approval}

For Guinea, approval for the investigations were obtained from the National Ethics Committee of Guinea (permit n²003/PFHG/05/GUI and 12/CNERS/ 12). For Tanzania, all the procedures followed the Animal Ethics guidelines as laid out by the Sokoine University of Agriculture (Morogoro, Tanzania).

\section{Publisher's Note}

Springer Nature remains neutral with regard to jurisdictional claims in published maps and institutional affiliations.

\section{Author details}

${ }^{1}$ Evolutionary Ecology Group, University of Antwerp, Antwerp, Belgium. ${ }^{2}$ Projet des Fièvre Hémorragiques en Guinée, Hôpital Donka, Conakry, Guinea. ${ }^{3}$ Bernhard-Nocht-Institute for Tropical Medicine, Hamburg, Germany. ${ }^{4}$ Institute of Vertebrate Biology, Research Facility Studenec, The Czech Academy of Sciences, Brno, Czech Republic. ${ }^{5}$ Department of Ecology and Evolutionary Biology, University of California, Los Angeles, USA. ${ }^{6}$ Department of Ecology and Evolutionary Biology, University of Arizona, Tucson, USA. 
${ }^{7}$ Department of Biology, University of Kinshasa, P.O. Box. 190, Kinshasa XI, Democratic Republic of the Congo.

\section{Received: 19 January 2017 Accepted: 19 April 2017} Published online: 27 April 2017

\section{References}

1. Monath TP, Newhouse VF, Kemp GE, Setzer HWCA. Lassa virus isolation from Mastomys natalensis rodents during an epidemic in Sierra Leone. Science. 1974;185:263-5.

2. Gryseels S, Rieger T, Oestereich L, Cuypers B, Borremans B, Makundi R, et al. Gairo virus, a novel arenavirus of the widespread Mastomys natalensis: Genetically divergent, but ecologically similar to Lassa and Morogoro viruses. Virology. 2015;476:249-56

3. Gryseels S, Baird S, Borremans B, Makundi R, Leirs H, Goüy de Bellocq J. When viruses don't go viral: the importance of host phylogeographic structure in the spatial spread of arenaviruses. PLoS Pathog. 2017;13:1.

4. Olayemi A, Obadare A, Oyeyiola A, Igbokwe J, Fasogbon A, Igbahenah F, et al. Arenavirus diversity and phylogeography of Mastomys natalensis rodents, Nigeria. Emerg Infect Dis. 2016;22:694-7.

5. Günther S, Hoofd G, Charrel R, Röser C, Becker-Ziaja B, Lloyd G, et al. Mopeia virus-related arenavirus in Natal multimammate mice, Morogoro, Tanzania. Emerg Infect Dis. 2009;15:2008-12.

6. Wulff $\mathrm{H}$, McIntosh BM, Hamner DB, Johnson KM. Isolation of an arenavirus closely related to Lassa virus from Mastomys natalensis in south-east Africa. Bull World Health Organ. 1977;55:441-4

7. Ishii A, Thomas Y, Moonga L, Nakamura I, Ohnuma A, Hang'ombe B, et al. Novel arenavirus, Zambia. Emerg Infect Dis. 2011;17:1921-4

8. McCormick JB. Lassa fever. In: Saluzzo JF, Dodet B, editors. Emergence and Control of Rodent-Borne Viral Diseases. Berlin: Elsevier; 1999. p. 177-95.

9. Monath TP. Lassa fever: New issues raised by field studies in West Africa. J Infect Dis. 1987;155:433-6.

10. World Health Organization: WHO fact sheet. http://www.who.int/csr/ disease/lassafever/en/). Accessed 23 Apr 2017.

11. Walker DH, Murphy FA. Pathology and pathogenesis of arenavirus infections. Curr Top Microbiol Immunol. 1987;133:89-113.

12. Günther S, Lenz O. Lassa virus. Crit Rev Clin Lab Sci. 2004;41:339-90.

13. Anderson RM, May R. Population biology of infectious diseases: Part II. Nature. 1979;280:361-7.

14. Anderson RM, May RM. Coevolution of host and parasites. Parasitology. 1982:85:411-26

15. Frank S. Models of parasite virulence. Q Rev Biol. 1996;71:37-78.

16. Alizon S, Hurford A, Mideo N, Van Baalen M. Virulence evolution and the trade-off hypothesis: history, current state of affairs and the future. J Evol Biol. 2009;22:245-59.

17. Knell RJ. Syphilis in renaissance Europe: rapid evolution of an introduced sexually transmitted disease? Proc Biol Sci. 2004;271(Suppl):S174-6.

18. Dwyer G, Levin S, Buttel L. A simulation model of the population dynamics and evolution of myxomatosis. Ecol. Monogr. 1990;60(4):423-47

19. Kerber R, Rieger T, Busch C, Flatz L, Pinschewer DD, Kümmerer BM, et al. Cross-species analysis of the replication complex of Old World arenaviruses reveals two nucleoprotein sites involved in $\mathrm{L}$ protein function. J Virol. 2011:85:12518-28.

20. Childs JE, Peters CJ. Ecology and epidemiology of arenaviruses and their hosts. In: Salvato MS, editor. The Arenaviridae. New York: Plenum Press; 1993. p. 331-85.

21. Oldstone M. Arenaviruses: biology and immunotherapy. In: Clarke P, editor. Current topics in microbiology and immunology. New York: Springer Verlag; 2002. p. 1-142.

22. Hotchin JE, Cinits M. Lymphocytic choriomeningitis infection of mice as a model for the study of latent virus infection1. Can J Microbiol. 1958;4:149-63.

23. Oldstone M, Dixon F. Susceptibility of different mouse strains to lymphocytic choriomeningitis virus. J Immunol. 1968;100:355-7.

24. Mims CA. Observations on mice infected congenitally or neonatally with lymphocytic choriomeningitis. Arch. Virol. 1970;30(1):67-74.

25. Oldstone $M$, Sinha $Y$, Blount $P$, Tishon $A$, Rodriguez $M$, von Wedel $R$, et al. Virus-induced alterations in homeostasis: Alterations in differentiated functions of infected cells in vivo. Science. 1982;218:6.

26. Webb $P$, Justines $G$, Johnson $K$. Infection of wild and laboratory animals with Machupo and Latino viruses. Bull World Health Organ. 1975;52:493-9.
27. Vitullo A, Hodara V, Merani MS. Effect of persistent infection with Junin virus on growth and reproduction of its natural reservoir, Calomys musculinus. Am J Trop Med Hyg. 1987;37:663-9.

28. Vitullo A, Merani MS. Vertical transmission of Junin virus in experimentally infected adult Calomys musculinus. Intervirology. 1990;31:339-44.

29. Borremans B, Vossen R, Becker-ziaja B, Gryseels S, Hughes N, Van Gestel M, et al. Shedding dynamics of Morogoro virus, an African arenavirus closely related to Lassa virus, in its natural reservoir host Mastomys natalensis. Sci Rep. 2015;5:10445.

30. Walker DH, Wulff H, Lange JV, Murphy FA. Comparative pathology of Lassa virus infection in monkeys, guinea-pigs, and Mastomys natalensis. Bull World Health Organ. 1975;52:523-34,

31. Demartini J, Green D, Monath TP. Lassa virus infection in Mastomys natalensis in Sierra Leone: Gross and microscopic findings in infected and uninfected animals. Bull World Health Organ. 1975;52:651-63.

32. Lalis A, Evin A, Janier M, Koivogui L, Denys C. Host evolution in Mastomys natalensis (Rodentia: Muridae): an integrative approach using geometric morphometrics and genetics. Integr Zool. 2015;10:505-14.

33. Leirs H, Stuyck J, Verhagen R, Verheyen W. Seasonal variation in growth of Mastomys natalensis (Rodentia: Muridae) in Morogoro, Tanzania. Afr J Ecol. 1990;28:298-306.

34. Demby AH, Inapogui A, Kargbo K, Koninga J, Kourouma K, Kanu J, et al. Lassa fever in Guinea: II. Distribution and prevalence of Lassa virus infection in small mammals. 2001;1:283-99.

35. Borremans B, Leirs H, Gryseels S, Günther S, Makundi R, Goüy de Bellocq J. Presence of Mopeia virus, an African arenavirus, related to biotope and individual rodent host characteristics: implications for virus transmission. Vector Borne Zoonotic Dis. 2011;11:1125-31.

36. Kallio ER, Helle H, Koskela E, Mappes T, Vapalahti O. Age-related effects of chronic hantavirus infection on female host fecundity. J Anim Ecol. 2015;84:1264-72.

37. Lord RD. The lens as an indicator of age in cottontail rabbits. J Wild Manage. 1959;23:358-60.

38. Morris P. A review of mammalian age determination methods. Mamm Rev. 1972;2:69-104.

39. Leirs H. Population ecology of Mastomys natalensis (Smith, 1834). Implications for rodent control in Africa. Brussels: Belgian Administration for Development Cooperation; 1995. Agricultural edition 35: 1994.

40. Augusteyn RC. Growth of the eye lens : I. Weight accumulation in multiple species. Mol Vis. 2014;20:410-26.

41. Jánová E, Nesvadbová J, Tkadlec E. Is the eye lens method of age estimation reliable in voles? Folia Zool. 2007:52:119-25.

42. Fichet-Calvet E, Lecompte E, Koivogui L, Soropogui B, Doré A, Kourouma F, et al. Fluctuation of abundance and Lassa virus prevalence in Mastomys natalensis in Guinea, West Africa. Vector Borne Zoonotic Dis. 2007;7:119-28.

43. Fichet-Calvet E, Lecompte E, Koivogui L, Daffis S, ter Meulen J. Reproductive characteristics of Mastomys natalensis and Lassa virus prevalence in Guinea, West Africa. Vector Borne Zoonotic Dis. 2008;8:41-8.

44. Fichet-Calvet E, Becker-Ziaja B, Koivogui L, Günther S. Lassa serology in natural populations of rodents and horizontal transmission. Vector Borne Zoonotic Dis. 2014:14:665-74.

45. Fichet-Calvet E, Ölschläger S, Strecker T, Koivogui L, Becker-Ziaja B, Camara $A B$, et al. Spatial and temporal evolution of Lassa virus in the natural host population in Upper Guinea. Sci Rep. 2016;6:1-6.

46. Vieth S, Drosten C, Lenz O, Vincent M, Omilabu S, Hass M, et al. RT-PCR assay for detection of Lassa virus and related Old World arenaviruses targeting the L gene. Trans R Soc Trop Med Hyg. 2007;101:1253-64.

47. Olschläger $S$, Lelke $M$, Emmerich $P$, Panning $M$, Drosten C, Hass $M$, et al. Improved detection of Lassa virus by reverse transcription-PCR targeting the 5' region of S RNA. J Clin Microbiol. 2010;48:2009-13.

48. Wulff $\mathrm{H}$, Lange J. Indirect immunofluorescence for the diagnosis of Lassa fever infection. Bull World Health Organ. 1975;52:429-36.

49. Hufert FT, Liidke W, Sehmitz H. Epitope mapping of the Lassa virus nucleoprotein using monoclonal anti-nucleocapsid antibodies. Arch Virol. 1989;106:201-12.

50. R Core Team. R: A language and environment for statistical computing. Vienna: R Foundation for Statistical Computing; 2016. http://www.r-project. org. Accessed 12 Dec.

51. Bernshtein A, Apekina N, Mikhailova T, Myasnikov Y, Khlyap L, Korotkov Y, et al. Dynamics of Puumala hantavirus infection in naturally infected bank voles (Clethrinomys glareolus). Arch Virol. 1999;144:2415-28. 
52. Verhagen R, Leirs H, Tkachenko E, van der Groen G. Ecological and epidemiological data on hantavirus in bank vole populations in Belgium. Arch Virol. 1986;91:193-205.

53. Kallio ER, Poikonen A, Vaheri A, Vapalahti O, Henttonen $H$, Koskela E, et al. Maternal antibodies postpone hantavirus infection and enhance individual breeding success. Proc Biol Sci. 2006;273:2771-6.

54. Kallio ER, Voutilainen L, Vapalahti O, Vaheri A, Henttonen $H$, Mappes T, et al. Endemic hantavirus infection impairs the winter survival of its rodent host. Ecology. 2007;88(8):1911-6.

55. Tersago K, Crespin L, Verhagen R, Leirs H. Impact of Puumala virus infection on maturation and survival in bank voles: a capture-mark-recapture analysis. J Wildl Dis. 2012;48:148-56.

56. Voutilainen L, Sironen T, Tonteri E, Tuiskunen Bäck A, Razzauti M, Karlsson M, et al. Life-long shedding of Puumala hantavirus in wild bank voles (Myodes glareolus). J Gen Virol. 2015:96:1238-47.

57. Barber I, Dingemanse NJ. Parasitism and the evolutionary ecology of animal personality. Philos Trans R Soc Lond B Biol Sci. 2010;365:4077-88.

58. Sluydts V, Crespin L, Davis S, Lima M, Leirs H. Survival and maturation rates of the African rodent, Mastomys natalensis: density-dependence and rainfall. Integr Zool. 2007;2:220-32.

59. Goyens J, Reijniers J, Borremans B, Leirs H. Density thresholds for Mopeia virus invasion and persistence in its host Mastomys natalensis. J Theor Biol. 2013;317:55-61.

60. Vitullo A, Merani M. Is vertical transmission sufficient to maintain Junin virus in nature? J Gen Virol. 1988;69:1437-40. 56. 57.

61. Veenstra AJF. The behaviour of the multimammate mouse, Rattus (Mastomys) natalensis (A. Smith). Anim Behav. 1958;6:195-206.

62. Kennis J, Sluydts V, Leirs H, van Hooft WFP. Polyandry and polygyny in an African rodent pest species, Mastomys natalensis. Mammalia. 2008;72:150-60.

63. Voutilainen L, Kallio ER, Niemimaa J, Vapalahti O, Henttonen H. Temporal dynamics of Puumala hantavirus infection in cyclic populations of bank voles. Sci Reports. 2016;6:1-14.

64. Sauvage F, Langlais M, Yoccoz NG, Pontier D. Modelling hantavirus in fluctuating populations of bank voles: the role of indirect transmission on virus persistence. J Anim Ecol. 2003;72:1-13.

65. Carlos J, Torre D, Oldstone M. Selective disruption of growth hormone transcription machinery by viral infection. Proc Natl Acad Sci U S A. 1992;89:9939-43.

66. Oldstone M, Southern P, Rodriquez M, Lampert P. Virus persists in beta cells of islets of Langerhans and is associated with chemical manifestations of diabetes. Science. 1984;224:1440-3.

\section{Submit your next manuscript to BioMed Central and we will help you at every step:}

- We accept pre-submission inquiries

- Our selector tool helps you to find the most relevant journal

- We provide round the clock customer support

- Convenient online submission

- Thorough peer review

- Inclusion in PubMed and all major indexing services

- Maximum visibility for your research

Submit your manuscript at www.biomedcentral.com/submit

CBiomed Central 